\title{
Möglichkeiten der rechtlichen Absicherung der innerparteilichen Willensbildung
}

Matthias Rossi, Augsburg

\section{A. Einleitung}

Wenn auf einem Symposion zur „(Partei-)Politik im Zeichen des Marketing“ nach den Möglichkeiten der rechtlichen Absicherung der innerparteilichen Willensbildung gefragt wird, ist zunächst zu skizzieren, welchen Einfluss Marketing auf die innerparteiliche Willensbildung haben kann. Diese Frage muss insbesondere deshalb geklärt werden, weil sich Marketing politischer Parteien - jedenfalls auf den ersten Blick - in erster Linie als ein Instrument im Verhältnis zwischen den Parteien und ihren Wählern darstellt, nicht dagegen so sehr als ein Instrument im Verhältnis zwischen einer Partei und ihren Mitgliedern. Insofern ist der Zusammenhang zwischen Marketing und innerparteilicher Willensbildung jedenfalls nicht offenkundig.

Begreift man Marketing jedoch generell als ein Instrument zur Steuerung der öffentlichen Meinung über die Medien und vergegenwärtigt man sich, dass Medien polarisieren, dramatisieren und akzelerieren, so wird deutlich, dass der zunehmende Einsatz von Marketing durch die politischen Parteien das Spannungsverhältnis verstärkt, das zwischen möglichst einheitlicher Außendarstellung auf der einen Seite und einem möglichst pluralistischen Meinungsbildungsprozess im Inneren besteht. Wer das Marketing bestimmt, bestimmt insoweit auch die Inhalte, möglicherweise vorbei an den an sich vorgesehenen Verfahren. Marketing verstärkt also jedenfalls dann, wenn es vom Parteivorstand eingesetzt wird, das Oligarchieproblem, das bereits Anfang des 20. Jahrhunderts erkannt und beschrieben wurde. ${ }^{1}$

$\mathrm{Ob}$ der Einsatz von modernem Marketing in Bezug auf die innerparteiliche Willensbildung die normative Kraft des Faktischen verstärkt oder ob vielmehr Morgensterns Hoffnung „Weil nicht sein kann, was nicht sein darf" gilt, soll im Folgenden in drei Schritten untersucht werden. Allen Ausführungen liegt dabei die folgende grundsätzliche Erkenntnis zu Grunde: Soweit rechtliche Absicherung zugleich eine staatliche Absicherung der innerparteilichen Willensbildung bedeutet, steht sie in einem Spannungsverhältnis zwischen der verfassungsrechtlich gewährleisteten Freiheit der politischen Parteien einerseits und dem verfassungsrechtlichen Gebot der innerparteilichen Demokratie. Darüber hinaus verlangt die rechtliche Absicherung der innerparteilichen Willensbildung nach einem hinreichend bestimmten inhaltli-

- Matthias Rossi ist Inhaber des Lehrstuhls für Staats- und Verwaltungsrecht, Europarecht sowie Gesetzgebungslehre an der Juristischen Fakultät der Universität Augsburg.

1 Vgl. M. Ostrogorski, La démocratie et l'organisation des partis politiques, 2 Bde, 1902; $R$. Michels, Zur Soziologie des Parteiwesens in der modernen Demokratie. Untersuchungen über die oligarchischen Tendenzen des Gruppenlebens, 1911, 4. Aufl. 1989. 
chen oder jedenfalls doch prozeduralen Maßstab. In diesem Sinne sollen unter (B.) zunächst die inhaltlichen Vorgaben an die innerparteiliche Willensbildung dargestellt werden, bevor unter (C.) die rechtliche Absicherung dieser binnendemokratischen Willensbildung untersucht wird. Ob über diesen Befund de lege lata hinaus weitere Sicherungsinstrumente de lege ferenda erforderlich und ggf. zulässig sind, soll abschließend unter (D.) untersucht werden.

\section{B. Inhaltliche Vorgaben an die innerparteiliche Willensbildung}

Die inhaltlichen Vorgaben an die innerparteiliche Willensbildung sind auf drei verschiedenen Ebenen normiert. Sie ergeben sich aus verfassungsrechtlichen Minimalanforderungen (I.), ihren gesetzlichen Konkretisierungen (II.) sowie vor allem aus der jeweiligen satzungsrechtlichen Ausgestaltung (III.).

\section{Verfassungsrechtliche Minimalanforderungen}

Die verfassungsrechtlichen Minimalanforderungen an die innerparteiliche Willensbildung ergeben sich aus Art. 21 Abs. 1 S. 3 GG: „Ihre innere Ordnung muss demokratischen Grundsätzen entsprechen." Bevor die Mindestgehalte dieses Verfassungssatzes näher betrachtet werden, muss zunächst seine Bedeutung erfasst werden.

\section{Bedeutung des Gebots innerparteilicher Demokratie}

Die verfassungsrechtlichen Anforderungen an die innerparteiliche Willensbildung stehen in einem Spannungsverhältnis zwischen der Staatsfreiheit der politischen Parteien und ihrer binnendemokratischen Organisation. Dieses Spannungsverhältnis ist verfassungsrechtlich nur grob aufgelöst: Einerseits ist die Freiheit der politischen Parteien vom Staat und von staatlichen Vorgaben in Bezug auf die Binnenorganisation durch diesen Satz von vornherein zurückgenommen. Die Verfassung lässt nicht jede innere Ordnung der politischen Parteien zu, auch wenn dies bei abstrakter Betrachtung zur Freiheit der politischen Parteien zählte, sondern verlangt, dass die innere Ordnung der politischen Parteien demokratischen Grundsätzen entspricht. Andererseits, und dies wird unter (C.) näher zu beleuchten sein, führt ein Verstoß gegen die innerdemokratische Organisation nicht per se zur Verfassungswidrigkeit Art. 21 Abs. 2 GG knüpft gerade nicht an Art. 21 Abs. $1 \mathrm{~S} .3 \mathrm{GG}$ an.

Die Auflösung dieses Spannungsverhältnisses ist Aufgabe des Gesetzgebers. Insoweit ist durchaus von Interesse, dass ursprünglich nur ein einfacher Gesetzesvorbehalt in Art. $21 \mathrm{GG}$ geplant war, so dass keine inhaltlichen Vorgaben für die Binnenorganisation der politischen Parteien aus der Verfassung erkennbar gewesen 
wären. ${ }^{2}$ Nicht der Herrenchiemsee-Entwurf, sondern erst ein vom Allgemeinen Redaktionsausschuss des Parlamentarischen Rates unterbreiteter Vorschlag ${ }^{3}$ führt mit der Regelung des Art. 21 Abs. 1 S. 3 GG zur ,erzwungenen Demokratisierung der politischen Parteien. ${ }^{.4}$ Der Sinn der jetzigen Vorgabe kann angesichts des berechtigen Wunsches der politischen Parteien nach klaren Linien und insbesondere nach einheitlicher Wahrnehmung durchaus hinterfragt werden. Er liegt jedenfalls weder in der Glaubwürdigkeitssicherung der politischen Parteien ${ }^{5}$ noch in einem Selbstzweck, sondern ist im Interesse des demokratischen Staates vorgeschrieben, der zwar nicht institutionell, aber doch personell mit Hilfe der Staaten gesteuert wird. ${ }^{6}$

Bei dem jetzigen qualifizierten Gesetzesvorbehalt ist eine funktionale Interpretation des Verfassungsgebots erforderlich. Bereits Konrad Hesse hat hervorgehoben, dass die innere Freiheit der Parteien gewährleistet wird, ,um auf diese Weise die Freiheit des politischen Prozesses an seiner Quelle zu sichern"?

$\mathrm{Ob}$ jedenfalls die verfassungsrechtliche Vorgabe an eine binnendemokratische Organisationsform tatsächlich notwendig ist oder ob nicht etwa die Grundrechte sowie die Gründungs- und Betätigungsfreiheit politischer Parteien genügen, um eine hinreichende Meinungsvielfalt und Chancengleichheit in den politischen Parteien sicherzustellen, mag dahingestellt bleiben. ${ }^{8}$ In jedem Fall ist die doppelte Relativierung zu beachten, die diese verfassungsunmittelbare Minimalanforderung charakterisiert: Zum einen wird die innere Ordnung eben nur demokratischen Grundsätzen unterworfen, deren Inhalt im Einzelnen eben nicht näher dargelegt werden. Zum anderen muss die innere Ordnung der politischen Parteien diesen Grundsätzen auch nur entsprechen, nicht dagegen mit ihnen identisch sein. Diese doppelte Relativierung ist vor allen Dingen deshalb sinnvoll, weil es kaum möglich ist, staatsfreie Partcien grundsätzlich denselben demokratischen Anforderungen zu unterwerfen, die für die Staatsorganisation und die politische Willensbildung in den Staatsorganen gelten. Insoweit geht Art. 21 Abs. 1 S. 3 GG zutreffend von einem Unterschied

2 Vgl. hierzu Rechtliche Ordnung des Parteiwesens, Bericht der vom Bundesminister des Innern eingesetzten Parteienrechtskommission, 2. Aufl. 1958, S. 155 f.; sowie H.H. Klein, in: Maunz/Dürig /(Hrsg.), GG, Art. 21 Rn. 112 ff.

3 Vorschlag v. 13. Dezember 1948; zitiert nach H.H. Klein, in: Maunz/Dürig /(Hrsg.), GG, Art. $21 \mathrm{Rn} .121$.

4 F. A. v. d. Heydte-Sacherl, Soziologie der deutschen Parteien, 1955, S. 153.

5 Stark kritisiert von $G$. Roellecke, in: Umbach/Clemens, GG, 2002, Art. 21 Rn. 79, nach dem sie „eine politisch-pādagogische Illusion aus dem Arsenal der Parteistaatsdoktrin ist.“

6 D. Grimm, in: Schneider/Zeh (Hrsg.). Parlamentsrecht und Parlamentspraxis in der Bundesrepublik Deutschland, 1989, §6 Rn. 20.

7 K. Hesse, Die verfassungsrechtliche Stellung der politischen Partei im modernen Staat, VVDStRL 17, S. 11, 30.

8 D. Grimm, in: Schneider/Zeh (Hrsg.), Parlamentsrecht und Parlamentspraxis in der Bundesrepublik Deutschland, $1989, \S 6 \mathrm{Rn}$. 4. erkennt in der Gründungsfreiheit zutreffend ein Korrektiv für die gestörte Rückkopplung politischer Parteien an vernachlässigte Bevölkerungsgruppen oder vernachlässigte Politikthemen. 
zwischen der Notwendigkeit demokratischer Strukturen im Staat und der in den (staatsfreien) politischen Parteien aus.

\section{Mindestgehalt}

Versucht man in Konkretisierung dieser doppelten Relativierung, die Mindestgehalte zu umschreiben, die sich aus Art. 21 Abs. 1 S. 3 GG ergeben und insoweit vom ausgestaltenden Gesetzgeber beachtet werden müssen, so lassen sich folgende Aspekte hervorheben:

In Anlehnung an den Grundsatz der Demokratie, der für den Staat in Art. 20 Abs. 2 S. 1 GG zum Ausdruck kommt ("Alle Staatsgewalt geht vom Volke aus."), lässt sich der Grundsatz festhalten, dass in politischen Parteien alle Entscheidungen auf die Mitglieder der politischen Parteien zurückzuführen sein müssen. Von entscheidender Bedeutung ist insoweit, dass die nach außen wirkenden Vertreter der politischen Parteien durch Wahlen bestimmt werden. Auch das Merkmal, dass Demokratie Herrschaft auf Zeit bedeutet, wird man auf politische Parteien übertragen können und deshalb eine Periodizität der Wahlen verlangen müssen. In solchen Wahlen (wic auch ggf. in Abstimmungen) ist es darüber hinaus ein demokratischer Grundsatz, dass Entscheidungen mit der Mehrheit getroffen werden, dass also das Mehrheitsprinzip gilt. Zugleich ist es der Demokratie auch im Sinne eines Grundsatzes immanent, dass Minderheiten hinreichend geschützt werden müssen, um - jedenfalls theoretisch - zur Mehrheit werden zu können. Insoweit sind Mindestanforderungen an den Minderheitenschutz verfassungsrechtlich vorgeschrieben. Demokratie ist darüber hinaus durch Transparenz gekennzeichnet. Übertragen auf die innere Ordnung der politischen Parteien wird man deshalb schon aus Art. 21 Abs. 1 S. 3 GG eine hinreichende Transparenz der Entscheidungsfindung innerhalb von politischen Parteien einschließlich innerparteilicher Informationsrechte verlangen müssen. Zu demokratischen Grundsätzen gehört es darüber hinaus auch, dass die Entscheidungsfindung nach rechtlich vorgegebenen Verfahren verläuft. Insoweit wird der Grundsatz der demokratischen Willensbildung ergänzt durch ein Minimum an rechtlicher Absicherung dieser Verfahren. ${ }^{9}$

Die entscheidende Frage ist schließlich, ob Art. 21 Abs. 1 S. 3 GG auch eine Willensbildung innerhalb der politischen Parteien von unten nach oben verlangt. Grundsätzlich wird dies in Literatur und Rechtsprechung angenommen. Allerdings hat bereits Konrad Hesse eine solche Willensbildung von unten nach oben als wirklich-

9 Vgl. zu den Mindestgehalten etwa M. Morlok, in: Dreier (Hrsg.), GG-Kommentar, Bd. 2. 2. Aufl. 2006, Art. 21 Rn. 125; Ch. Gusy, in: AK-GG, 3. Aufl. 2001, Art. 21 Rn. 67 ff. Instruktiv auch $U$. v. Alemann, Mehr Demokratie per Dekret? Innerparteiliche Auswirkungen des Deutschen Parteiengesetzes von 1967, in: Politische Vierteljahresschrift, Bd. 13, H. 2, S. $181,184 \mathrm{ff}$. 
keitsfremd bezeichnet ${ }^{10}$ und etwa Christoph Gusy als viel zu eindimensional erkannt. ${ }^{11}$ Bei tatsächlicher Betrachtung erscheint in jedem Fall fraglich, ob der Wille wirklich unten gebildet oder nicht nur von unten bestätigt wird. In jedem Fall dürfen die verfassungsrechtlichen Anforderungen an eine solche Willensbildung nicht überspannt werden. Während es grundsätzlich zunächst Sache des einfachen Gesetzgebers bleibt, diese Verfassungsregel näher auszugestalten, sind es darüber hinaus vor allen Dingen die politischen Parteien, die über ihre interne Willensbildung in den Satzungen entscheiden und sich dadurch zugleich ein bestimmtes Profil geben können. Nicht übersehen werden darf in jedem Fall, und dies ist im Rahmen eines Symposions zum Marketing der politischen Parteien von besonderer Bedeutung, dass eine zu ausgeprägte basisdemokratische Binnenstruktur dem Wunsch und der Notwendigkeit entgegenstehen kann, nach außen mit einer Stimme zu sprechen und sich mit markanten Slogans und Bildern zu verkaufen. Vergleicht man bei aller Vorsicht die Ausgestaltung der staatlichen Demokratie mit den Anforderungen an die innerparteiliche Demokratie, so ist nicht zu übersehen, dass auch der Staat nach außen eher mit einer Stimme spricht, dass hier über Art. 32 Abs. 1 GG und Art. 59 Abs. 1 GG die Befugnisse in erster Linie beim Bund und nicht etwa bei den Ländern und darüber hinaus noch beim Bundespräsidenten bzw. der Bundesregierung und nicht etwa beim Bundestag und Bundesrat liegen. Eine basisorientierte politische Willensbildung mag in ruhigen Zeiten, in denen das Schiff im Hafen liegt, sinnvoll und möglich sein, in rauhen Zeiten, auf hoher, stürmischer See wird dagegen häufig der Kapitän Anweisungen treffen und sich dafür dann erst hinterher verantworten müssen. Die Entscheidungsfindung, die Willensbildung, erfolgt insoweit nicht immer vor einer Maßnahme, sondern ggf. erst hinterher. Dieses bestätigende - auf staatlicher Ebene würde man sagen legitimierende - Element mag durchaus ausreichen, um den verfassungsrechtlichen Anforderungen an die parteipolitische Willensbildung zu entsprechen.

\section{Gesetzliche Konkretisierungen}

Gesetzliche Konkretisierungen des Art. 21 Abs. 1 S. 3 GG finden sich vor allen Dingen im Parteiengesetz (1.), in einigen Spezialvorschriften des Bundeswahlgesetzes (2.) sowie in subsidiär zur Anwendung kommenden Vorgaben des Bürgerlichen Gesetzbuchs (3.).

$10 K$. Hesse, Die verfassungsrechtliche Stellung der politischen Partei im modernen Staat, VVDStRL 17, S. 11, 21.

11 Ch. Gusy, in: AK-GG, 3. Aufl. 2001, Art. 21 Rn. 67. 
Das Parteiengesetz enthält vielerlei Vorschriften, die als Konkretisierung des binnendemokratischen Organisationsgebots des Art. 21 Abs. 1 S. 3 GG verstanden werden können. $\mathrm{Zu}$ beachten ist bei der folgenden Aufzählung der einzelnen Normen, dass sich die Darstellung am geltenden Parteiengesetz orientiert. Andere gesetzliche Ausgestaltungen sind insofern durchaus denkbar, ist der Gestaltungsspielraum, den Art. 21 Abs. 1 S. 3 GG dem Gesetzgeber lässt, doch sehr weit.

a) Satzung und Programm der politischen Parteien

\$6 Abs. 1 PartG schreibt zunächst vor, dass politische Parteien über eine schriftliche Satzung und ein schriftliches Programm verfügen müssen. Von Interesse für das Thema der binnendemokratischen Organisation ist vor allem die Pflicht, eine schriftliche Satzung zu fixieren. Denn während das politische Programm vor allen Dingen die inhaltlichen Zielsetzungen der politischen Partei zum Ausdruck bringen soll, ist es Aufgabe der Satzungen, die in $\S 6$ Abs. 2 PartG verankerten Mindestvorgaben zu bestimmen, die ihrerseits sämtlich auf die Organisation der Partei hinauslaufen. Die Vorgabe, eine binnendemokratische Organisation zu wählen, wird insoweit in $\$ 6$ Abs. 2 PartG konkretisiert.

\section{b) Gliederung der politischen Parteien}

Gegenstand dieser Satzung muss nach $\S 6$ Abs. 2 Nr. 6 PartG unter anderem die allgemeine Gliederung der Parteien sein. Dies wir näher konkretisiert in $\S 7$ PartG. Die Norm verlangt unter anderem, dass sich die politischen Parteien in Gebietsverbände gliedern. Was auf den ersten Blick eher als Ausprägung eines föderativen denn als eines demokratischen Prinzips erscheint, hat seine Wurzeln und Ziele doch zugleich auch in der Realisierung des binnendemokratischen Organisationsauftrags des Art. 21 Abs. 1 S. 3 GG. Denn wie auch auf staatlicher Ebene einer der Vorteile der föderativen Gliederung darin liegt, dass das demokratische Element durch die Zuordnung zu einer kleineren Bevölkerungsgruppe gestärkt wird, so gilt dieser Gedanke auch für politische Parteien und kommt dazu ausdrücklich in $\S 7$ Abs. $1 \mathrm{~S} .3$ PartG zum Ausdruck: „Die gebietliche Gliederung muss so weit ausgebaut sein, dass den einzelnen Mitgliedern eine angemessene Mitwirkung an der Willensbildung der Partei möglich ist.“ 
c) Mitgliederversammlung und Organe

Die grundsätzliche Satzungsautonomie der politischen Parteien wird durch § 6 PartG weiterhin dadurch eingeschränkt, dass jede politische Partei über bestimmte Organe verfügen muss und darüber hinaus Mitgliederversammlungen einberufen und durchführen muss. Zu den von $\S 9$ PartG vorgesehenen obligatorischen Organen zählt in erster Linie die Mitgliederversammlung, darüber hinaus aber auch der Vorstand. Andere Organe einzurichten unterliegt der Gestaltungsautonomie jeder einzelnen politischen Partei.

Von besonderer Bedeutung für die demokratische Binnenstruktur der politischen Parteien ist das Organ der Mitgliederversammlung, das synonym auch als Vertreterversammlung, Parteitag oder Hauptversammlung bezeichnet wird. In Umsetzung des demokratischen Grundsatzes einer Herrschaft auf Zeit und zur Absicherung einer hinreichenden Einflussnahme der Parteimitglieder auf personelle und inhaltliche Ausrichtungen der politischen Parteien sieht $\S 9$ Abs. 1 S. 3 PartG vor, dass die Parteitage mindestens zweijährlich zusammentreten müssen. Die legitimations- bzw. vollmachtserteilende Macht der Mitgliederversammlungen kommt darüber hinaus in $\S 9$ Abs. 4 PartG zum Ausdruck, nach dem die Mitgliederversammlung den Vorstand wählen muss. Zugleich kann es als ein Element des Demokratieprinzips betrachtet werden, dass dieser Vorstand kraft gesetzlicher Regelung des $\$ 9$ Abs. 4 PartG ein Kollegialorgan darstelit, also aus mehreren Personen besteht, so dass insoweit eine monarchisch ausgerichtete Führerschaft einer einzelnen Person rechtlich - nicht faktisch - ausgeschlossen ist. ${ }^{12}$

\section{d) Rechte der Mitglieder}

Eine Subjektivierung der demokratischen Binnenorganisation erfolgt in $\S 10$ PartG. Diese Vorschrift verleiht den Mitgliedern politischer Parteien - wenige - Rechte. Zu nennen ist zunächst das Recht, einer politischen Partei beizutreten. Dieses Recht ist freilich nur schwach ausgestaltet, muss doch die Ablehnung eines Aufnahmeantrags nach $\S 10$ Abs. 1 S. 2 PartG nicht begründet werden. Gleichwohl verlangt das Parteiengesetz schon hier eine generelle Öffnung der im gesellschaftlichen Leben verwurzelten Parteien, als in $\S 10$ Abs. 1 S. 3 allgemeine, auch befristete Aufnahmesperren nicht zulässig sind. Dies kann und mag als Ausprägung des Minderheitenschutzes verstanden werden, soll jedenfalls sicherstellen, dass politische Parteien nicht zum „closed shop“ werden, sondern sich immer wieder neu der gesellschaftlichen Veränderung stellen müssen. ${ }^{13}$

12 Diesen Zusammenhang erwähnend H. Wißmann, in: Kersten/Rixen (Hrsg.), PartG, 2009, § 11 Rn. 4.

13 Näher hierzu, insb. zur Frage, ob aus der Norm ein Aufnahmeanspruch folgt, S. C. Lenski, in: Rossi/Lenski, PartG. 2010. §11 Rn. 9 ff. 
Noch deutlicher als Ausdruck des demokratischen Binnenorganisationsgrundsatzes ist die Gewährleistung des gleichen Stimmrechts aller Mitglieder. In Anlehnung an den Wahlrechtsgrundsatz der Gleichheit, wie er in Art. 38 GG für die Wahlen zum Deutschen Bundestag angeordnet ist und über Art. 28 Abs. 1 S. 2 GG auch für die Länder gilt, wird hier die grundsätzliche Gleichheit aller Mitglieder gesetzlich normiert und insoweit satzungsrechtlichen Ausgestaltungen und Differenzierungen entzogen. Freilich gilt dieses gleiche Stimmrecht nicht unbeschränkt, sondern kann unter anderem von der Zahlung der Beitragspflicht abhängig gemacht werden. ${ }^{14}$

Als letztes Recht sieht $\S 10$ Abs. 2 S. 3 PartG die Möglichkeit eines jeden Mitglieds vor, jederzeit aus einer Partei auszutreten.

Weitere Rechte sind dagegen im Parteiengesetz nicht verankert. Sie sind insoweit der satzungsrechtlichen Ausgestaltung und damit den einzelnen politischen Parteien überlassen.

\section{e) Zusammensetzung des Vorstands}

Als Konkretisierung der binnendemokratischen Organisation politischer Parteien kann weiterhin auch $\S 12$ Abs. 2 S. 2 PartG verstanden werden. Nach dieser Vorschrift darf der Anteil der nicht gewählten Mitglieder ein Drittel der Gesamtmitgliederzahl des Organs nicht übersteigen. Vorstandsmitglieder dürfen mit anderen Worten nicht mehr als ein Drittel der Mitglieder in den allgemeinen Parteiausschüssen stellen. Vergegenwärtigt man sich die Bedeutung der Parteiausschüsse, die durchaus verglichen werden können mit den Ausschüssen staatlicher Organe, wird deutlich, dass mit dieser quantitativen Beschränkung der Einfluss weniger Personen auf die Willensbildung und -betätigung innerhalb der politischen Parteien begrenzt werden soll. Freilich bleibt diese Regelung wie viele andere Regelungen im Parteiengesetz auch insoweit an der Oberfläche, als dass eben nur eine quantitative Begrenzung vorgenommen werden kann. Dem faktischen Einfluss einiger weniger Mitglieder, die - sei es qua Natur, sei es qua Amt - über besondere Autorität verfügen und deshalb die Entscheidungsfindung auch in den allgemeinen Parteiausschüssen in besonderer Art und Weise zu beeinflussen vermögen, kann das Recht nichts entgegen setzen.

\section{f) Willensbildung in den Organen}

Hinsichtlich der Willensbildung in den Organen schreibt § 15 PartG Mindestanforderungen vor, die von den politischen Parteien in ihren Satzungen nicht unterschritten werden dürfen. Vier Aspekte seien herausgegriffen: 
Zunächst schreibt § 15 Abs. 1 PartG ganz grundsätzlich das Mehrheitsprinzip fest, entschieden wird danach grundsätzlich mit relativer Mehrheit. Hinzuweisen ist bereits an dieser Stelle darauf, dass andere Mehrheitsverhältnisse durchaus in Betracht kommen und beachtet werden müssen, wenn sie gesetzlich verankert sind. Eine besondere Rolle spielt insoweit $\S 33$ Abs. I S. 2 BGB, der für Satzungsänderungen eine qualifizierte relative Mehrheit vorsieht.

$\S 15$ Abs. 2 PartG normiert darüber hinaus den Grundsatz der Geheimheit von Wahlen. Auch dieser Grundsatz kann als Schutz der Minderheit verstanden werden und ist ausgeprägtes Merkmal einer Demokratie. Freilich kommt $\$ 15$ Abs. 2 S. 2 PartG dem Erfordernis der Praxis insoweit entgegen, als dass solange offen abgestimmt werden kann, solange sich dagegen kein Widerstand regt.

Der politische Einfluss eines einzelnen Mitglieds wird darüber hinaus auch durch $\S 15$ Abs. 3 S. 1 PartG gesichert. Danach ist das Antragsrecht so zu gestalten, dass eine demokratische Willensbildung gewährleistet bleibt, insbesondere auch Minderheiten ihre Vorschläge ausreichend zur Erörterung bringen können. ${ }^{15}$

Schließlich sei auch auf $\S 15$ Abs. 3 S. 3 PartG hingewiesen. Nach dieser Vorschrift ist eine Bindung an Beschlüsse anderer Organe bei Wahlen und Abstimmungen unzulässig.

\section{g) Parteischiedsgerichte}

$\S 14$ PartG verlangt, dass die politischen Parteien Parteischiedsgerichte einrichten. Hier wird der demokratische Organisationsvorbehalt des Art. 21 Abs. 1 S. 3 GG in rechtlicher Hinsicht prozedural gesichert. Parteischiedsgerichte können essentielle Bedeutung für den Schutz der einzelnen Mitgliederrechte erlangen und insoweit eine innerparteiliche Demokratie erst gewährleisten.

h) Aufstellung von Wahlbewerbern

In Fortschreibung des $\S 15$ Abs. 1 S. 2 PartG bestimmt auch $\S 17$ PartG, dass die Kandidaten für Wahlen zur Volksvertretung in geheimer Abstimmung nominiert werden müssen. Im Unterschied freilich zu § 15 PartG gilt dieser Grundsatz der Geheimheit an dieser Stelle absolut - Ausnahmen sind nicht zulässig. Die besondere Anforderung liegt darin begründet, dass mit der Aufstellung von Bewerbern die Schnittstelle zwischen der politischen Meinungsbildung innerhalb von politischen Parteien und ihrem Einfluss in staatlichen Organen betreten ist. 


\section{Spezialvorschriften im Bundeswahlgesetz}

An dieser Schnittstelle zwischen den politischen Parteien und ihrem Einfluss über staatliche Organe auf die staatliche Willensbildung steht das Bundeswahlgesetz. Diesbezüglich normiert der Vierte Abschnitt in den $\$ \$ 16$ ff. die Vorbereitung der Wahl, darunter auch in $\$ 21$ die Aufstellung von Wahlbewerbern. In Bezug auf das binnendemokratische Organisationsprinzip der politischen Parteien ist dabei insbesondere $\S 21$ Abs. 3 S. 2 BWahlG von Bedeutung: „Jeder stimmberechtigte Teilnehmer der Versammlung ist hierbei vorschlagsberechtigt." Die Vorschrift ist insoweit lex specialis gegenüber $\S 15$ Abs. 3 PartG, der das allgemeine Antragsrecht betrifft. In Bezug auf die Nominierung der Parteimitglieder für Parlamente gewinnt das demokratische Antragsrecht insoweit an besonderer Bedeutung und lässt sich als besonderer Schutz der politischen Minderheiten innerhalb einer politischen Partei verstehen.

\section{Subsidiäre Vorgaben des Bürgerlichen Gesetzbuchs}

Als in der Gesellschaft wurzelnde Personenzusammenschlüsse unterliegt das Organisationsverhalten der politischen Parteien im Übrigen den Vorschriften des BGB, die freilich ihrerseits im Lichte des Gebots innerparteilicher Demokratie ausgelegt werden müssen. ${ }^{16}$ Unabhängig von ihrer Organisation als rechtsfähige oder nichtrechtsfăhige Vereine ${ }^{17}$ haben die Parteien insbesondere die $\$ \S 21 \mathrm{ff}$. BGB zu beachten, die u.a. qualifizierte Mehrheitserfordernisse für bestimmte Entscheidungen festlegen. Besondere Bedeutung erlangt $\S 33$ Abs. 1 S. 2 BGB, der für Satzungsänderungen eine qualifizierte relative Mehrheit, nämlich die Mehrheit von drei Vierteln der anwesenden Mitglieder, verlangt. Diese Vorschrift ist ein anschauliches Beispiel dafür, dass sich der Inhalt der ,demokratischen Grundsätze“, denen die innere Organisation der politischen Parteien nach Art. 21 Abs. 1 S. 3 GG entsprechen muss, nicht strikt aus einer Übertragung der für die Staatsorganisation grundgesetzlich verfassten Demokratie ermitteln lässt. Wäre dies der Fall, ließe sich in Anlehnung an die für Verfassungsänderungen zu beachtenden Mehrheitserfordemisse des Art. 79 Abs. 2 GG daran denken, für Satzungsänderungen politischer Parteien die Zustimmung von zwei Dritteln aller Mitglieder zu verlangen - ein Erfordernis, das nicht praktikabel und wohl auch nicht praktizierbar wäre. Insofern sei noch einmal betont, dass die innere Organisation der politischen Parteien nicht wie die vom Grundgesetz verfasste staatliche Organisation ausgestaltet sein muss, sondern demokratischen Grundsätzen eben nur entsprechen muss.

16 S.-C. Lenski, in: Rossi/Lenski, PartG, 2010, § 15 Rn. 1.

17 Vgl. zur weitgehend Gleichbehandlung BGH, Urt. v. 2. Juli 2007 - II ZR 111/05, ZJS 2008

S. $64 \mathrm{~m}$. Anmerkung von C. Meller-Hannich. 


\section{Satzungsrechtliche Ausgestaltung}

Unter Beachtung der verfassungsrechtlichen Vorgaben und ihrer einfachgesetzlichen Konkretisierungen sind es vor allem die jeweiligen Satzungen der einzelnen politischen Parteien, die die rechtlichen Vorgaben für die innerparteiliche Willensbildung fixieren. In dieser zur Satzungsautonomie der politischen Parteien konkretisierten Privatautonomie kommt der Grundsatz der Staatsfreiheit der politischen Parteien besonders deutlich zum Ausdruck, wird ihre Verwurzelung im gesellschaftlichen Bereich rechtlich respektiert. Die weitgehende Satzungsautonomie gibt den politischen Parteien die Freiheit und Möglichkeit, sich nicht nur durch unterschiedliche Inhalte, sondern auch durch unterschiedliche Verfahren der internen Willensbildung und Beschlussfassung zu profilieren.

In Wahmehmung dieser Freiheit unterscheiden sich die Satzungen der einzelnen Parteien zum Teil erheblich. In den Satzungen der fünf im Bundestag vertretenen politischen Parteien lassen sich etwa Unterschiede hinsichtlich der Zusammensetzung des Bundesparteitags, der Beschlussfähigkeit, der Mehrheitserfordernisse bei Abstimmungen, der Gicltung von Wahlrechtsgrundsätzen und dem anzuwendenden Wahlsystem bei Personalwahlen, der Weisungsrechte im vertikalen Aufbau der politischen Parteien sowie hinsichtlich der Zulässigkeit, dem Inhalt und den Voraussetzungen eines Mitgliederentscheids ausmachen.

\section{Rechtliche Absicherung der innerparteilichen Willensbildung}

Die rechtlichen Vorgaben für die innerparteiliche Willensbildung können als normative Sollensbestimmungen die Realität nur in dem Maße gestalten, in dem ihre Missachtung entsprechende Wirkungen hervorruft (I.), die ihrerseits auch geltend gemacht und durchgesetzt werden können (II.). Rechtlich abgesichert ist aber auch dann nur die Einhaltung der normativen Vorgaben für die innerparteiliche Willensbildung, nicht hingegen die tatsächliche innerparteiliche Willensbildung, wie das vorgegebene Thema dieses Beitrags möglicherweise nahe legt.

\section{Wirkungen von Verstößen}

In einem hierarchisch gestuften Regelungswerk bildet die jeweils höhere Ebene den Maßstab für die Beurteilung der Rechtmäßigkeit der nachrangigen Ebenen. Allerdings scheidet Art. 21 Abs. 1 S. 3 GG wegen seiner Unbestimmtheit und des daraus folgenden gesetzlichen Konkretisierungsbedarfs als unmittelbarer Maßstab für die Beurteilung der binnendemokratischen Willensbildung grundsätzlich aus. Von zentraler Bedeutung für die rechtliche Beurteilung der abstrakt-generellen Satzungen sind vielmehr die skizzierten gesetzlichen Vorschriften, deren Missachtung zur Nichtigkeit von Satzungsbestimmungen führen kann (1.). Die Satzungen 
bilden ihrerseits den Maßstab für die rechtliche Beurteilung der konkıeten Beschlüsse der Organe politischer Parteien, die unwirksam sein können, wenn sie auf nichtigen Satzungen beruhen oder unter Verletzung entsprechender Satzungsbestimmungen gefasst werden (2.). Über diese Wirkungen der Nichtigkeit und Unwirksamkeit hinaus führen Verstöße gegen die gesetzlichen Vorschriften oder gar gegen Art. 21 Abs. 1 S. 3 GG aber weder zur automatischen Qualifizierung der politischen Partei als verfassungswidrig (3.) noch zum Verlust ihrer Parteieigenschaft (4.).

\section{Nichtigkeit von Satzungen}

Der Verstoß von Vorschriften einer Satzung gegen das Parteiengesetz oder andere zwingende Rechtsvorschriften führt zu ihrer Nichtigkeit. An dieser rechtlichen Wirkung bestehen keine Zweifel. ${ }^{18}$ Diskutiert wird nur, wie die Nichtigkeit der entsprechenden Satzungsbestimmungen zu begründen ist. Einerseits werden die gesetzlichen Bestimmungen als Verbotsgesetze im Sinne des § 134 BGB verstanden, so dass đie Nichtigkeit aus dieser Vorschrift begründet wird. ${ }^{19}$ Andererseits wird unmittelbar auf Art. 21 Abs. I S. 3 GG rekurriert und die Nichtigkeit als allgemeine Rechtsfolge für verfassungswidrige Normen abgeleitet. ${ }^{20}$ Gegen letztere Auffassung spricht zwar, dass Art. 21 Abs. 1 S. 3 GG inhaltlich zu unbestimmt ist, um als direkter Maßstab für die Ausgestaltung für Satzungen politischer Parteien zu fungieren. Erforderlich ist vielmehr, wie gezeigt, eine gesetzliche Ausgestaltung, so dass diese grundsätzlich auch den Maßstab für und die Rechtsfolge von Verletzungen darstellen muss. Gleichwohl bedarf es des Rückgriffs auf $\$ 134$ BGB und der Charakterisierung der Vorschriften des Parteiengesetzes und des Bundeswahlgesetzes als Verbotsgesetze nicht, denn die Nichtigkeit der diese Gesetze missachtenden Satzungsbestimmungen folgt als unmittelbare Konsequenz der Kollisionsregel "lex superior derogat legi inferiori" bereits aus dem allgemeinen Geltungsanspruch höherrangigen Rechts.

Unabhängig von der konkreten Begründung ist zu betonen, dass der Verstoß einer Satzungsbestimmung gegen höherrangiges Recht nicht zur Nichtigkeit der gesamten Satzung, sondern nur zur Nichtigkeit der jeweiligen Bestimmung führt. ${ }^{21}$ Dies folgt nicht nur aus dem allgemeinen Grundsatz, autonom gesetztes Recht soweit wie möglich in Kraft zu lassen und das Nichtigkeitsverdikt auf die Teile zu beschränken, die die Rechtsverletzung begründen, sonđern im Recht der politischen Parteien darüber hinaus erneut auch aus der (Staats-)Freiheit der politischen Parteien und ihrer Sat-

18 G. Roellecke, in: Umbach/Clemens, GG, 2002, An. 21 Rn. 40.

19 Vgl. J. Ipsen, in: Sachs (Hrsg.), GG, Art. 21 Rn. 86; Jarass/Pieroth, Art. 21 Rn. 26; P. Kunig, in: v. Münch/Kunig (Hrsg.) GG, Bd. II, Art. 21 Rn. 56.

20 So etwa M. Morlok, in: Dreier (Hrsg), GG-Kommentar, Bd. 2, 2. Aufl. 2006, Att. 21 Rn. 127; W. Henke, in: BK GG, Art. 21 Rn. 260.

21 A.A. VGH Kassel, NJW 1989, S. 2706; BVerwG, NJW 1989, S. 3297; W. Henke, Das Rechı det politischen Parteien, 2. Aufl. 1972, S, 58 f. 
zungsautonomie. Für eine weite Anwendung des $§ 139$ BGB ist insoweit kein Raum. An die Stelle der fehlerhaften Satzungsbestimmung tritt - soweit möglich - vielmehr die gesetzliche Regelung. Im Übrigen ist es an den Parteimitgliedern bzw. an den Wählern, die Verletzung gesetzlicher Vorschriften politisch zu sanktionieren. ${ }^{22}$

\section{Unwirksamkeit von Beschlüssen}

Ebenso sind Beschlüsse der Organe politischer Parteien unwirksam, wenn sie auf nichtigen Satzungen beruhen oder unter Verletzung der entsprechenden Satzungsbestimmungen gefasst werden. ${ }^{23}$ Auch dies folgt bereits aus der Maßstabswirkung höherrangigen Rechts, so dass es des Rückgriffs auf $\S 134$ BGB oder auf Art. 21 Abs. 1 S. 3 GG nicht zwingend bedarf.

\section{Keine zwingende Verfassungswidrigkeit}

Unter keinen Umständen folgt aus der Verletzung gesetzlicher Vorschriften durch Satzungen politischer Parteien oder gar aus einem satzungswidrigen Beschluss eines Organs einer politischen Partei ihre automatische Qualifizierung als verfassungswidrig i.S.d. Art. 21 Abs. 2 GG. Dies mag auf den ersten Blick als inkonsequent erscheinen, weil die Verletzung einer Verfassungsbestimmung (bzw. der sie konkretisierenden gesetzlichen Vorschriften) doch regelmäßig das Verdikt der Verfassungswidrigkeit nach sich zieht. Doch eine Interpretation der Vorschrift nach allen Auslegungsmethoden zeigt deutlich, dass das Gebot binnendemokratischer Parteiorganisation nicht mit der drohenden Verfassungswidrigkeit sanktioniert ist.

Dic Einstufung einer politischen Partei als verfassungswidrig unterliegt hohen materiellen und prozeduralen Anforderungen, die in Art. 21 Abs. 2 GG sowie in $\S \S 43 \mathrm{ff}$. BVerfGG abschließend geregelt sind. Bereits die Normierung dieser Voraussetzungen im eigenständigen Absatz 2 des Art. $21 \mathrm{GG}$ zeigt bei systematischer Betrachtung, dass das Verdikt der Verfassungswidrigkeit nicht an die Voraussetzungen des Art. 21 Abs. 1 GG anknüpft, die neben dem Gebot binnendemokratischer Organisation mit der Rechenschaftspflicht durchaus weitere organisationsbezogene Vorgaben für politische Parteien enthält. Vielmehr beschreibt Art. 21 Abs. 2 S. 1 GG, wie der klare Wortlaut zeigt, einen eigenständigen materiellen Maßstab für die Beurtcilung einer politischen Partei als verfassungswidrig. Nur „Parteien, die nach ihren Zielen oder nach dem Verhalten ihrer Anhänger darauf ausgehen, die freiheitliche demokratische Grundordnung zu beeinträchtigen oder zu beseitigen. sind verfassungswidrig." Die Verletzung derjenigen Vorschriften, die eine binnendemokratische Organisation der politischen Parteien sicherstellen wollen, ist dagegen kein

Vgl. J. Ipsen, in: Sachs (Hrsg.), GG, Art. 21 Rn. 88. 
Tatbestandsmerkmal dieser abschließenden und teleologisch eng auszulegenden Vorschrift. Insofern mag ein Verstoß gegen das in Art. 21 Abs. 1 S. 3 GG normierte Gebot binnendemokratischer Organisation die Verfassungsfeindlichkeit der politischen Partei nach Art. 21 Abs. 2 S. 1 GG indizieren, kann jedoch nicht von der exakten Prüfung dessen materieller Voraussetzungen befreien. Eine Partei kann entsprechend demokratischen Grundsätzen organisiert sein und doch verfassungsfeindliche Ziele verfolgen. Ungekehrt muss eine Partei, die nicht entsprechend demokratischen Grundsätzen organisiert ist, nicht zwangsläufig verfassungsfeindliche Ziele im Sinne des Art. 21 Abs. 2 GG verfolgen.

Im Übrigen statuiert Art. 21 Abs. 2 S. 2 GG in prozeduraler Hinsicht ein Feststellungsmonopol des Bundesverfassungsgerichts, das nach der geltenden Ausgestaltung des Bundesverfassungsgerichtsgesetzes besonders hohen Hürden unterworfen ist - die Verfassungswidrigkeit einer politischen Partei kann nach $\$ 15$ Abs. 4 S. 1 BVerfGG nur mit zwei Dritteln der Mitglieder des Senats festgestellt werden - ein Quorum, das bei acht Mitgliedern des Senats faktisch sogar einer Mehrheit von drei Vierteln entspricht. Bis zu einer solchen Feststellung bleibt es auch für eine politische Partei, die die gesetzlichen Anforderungen an die binnendemokratische Organisation in ihrer Satzung oder bei einer Beschlussfassung verletzt, bei der Vermutung ihrer Verfassungsmäßigkeit.

\section{Kein Verlust der Parteieigenschaft}

Die Missachtung von Vorschriften, die die binnendemokratische Organisation politischer Parteien sicherstellen wollen, führt auch nicht zum Verlust der Parteieigenschaft. Die in $\$ 2$ Abs. 1 ParG niedergelegte Definition einer politischen Partei knüpft nicht an die Einhaltung der in Rede stehenden Vorschriften an. Im Sinne der verfassungsrechtlich von Art. 21 Abs. 1 S. 2 GG betonten Gründungsfreiheit und einer daraus abzuleitenden Pluralität politischer Parteien beschränkt sich die Bestimmung vielmehr auf den Nachweis, dass eine Vereinigung von Bürgern mit einer gewissen organisatorischen Festigkeit Einfluss auf die politische Willensbildung und parlamentarische Vertretung im Bund oder in einem Land nehmen will und eine ausreichende Emsthaftigkeit dieser Ziele nach außen erkennbar ist.

Abgesehen davon, dass eine demokratischen Grundsätzen entsprechende Organisationsform somit nicht zu den tatbestandlichen Voraussetzungen des $\$ 2$ Abs. 1 PartG zählt, erschöpft sich đie Bedeutung des $\$ 2$ PartG auch für die (erstmalige) Gründung einer Partei und ihrer Zulassung durch den Wahlausschuss nach $\S 18$ Abs. 4 BWahlG. Ein darüber hinausgehendes Zulassungs- oder Registrierungsverfahren ist mit Rücksicht auf die Gründungsfreiheit politischer Partejen nicht vorgesehen und wäre wohl auch nicht zulässig. ${ }^{24}$ Jedenfalls kann dem $\$ 2$ Abs. 1 PartG nicht entnommen werden, dass eine existierende politische Partei ihre Parteieigen- 
schaft verliert, wenn sie die gesetzlichen Bestimmungen zur Sicherstellung einer binnendemokratischen Organisation missachtet. Es gibt vielmehr nur zwei Gründe, aus denen eine politische Partei ihre (rechtliche) Eigenschaft als solche verliert: $\S 2$ Abs. 2 PartG knüpft an das Emsthaftigkeitskriterium des $\S 2$ Abs. 1 PartG an und spricht den Verlust der Rechtsstellung einer politischen Partei aus, wenn sie sechs Jahre lang weder an einer Bundestagswahl noch an einer Landtagswahl mit eigenen Wahlvorschlägen teilgenommen hat. Darüber hinaus führt die Feststellung der Verfassungswidrigkeit einer politischen Partei durch das Bundesverfassungsgericht nach $\S 21$ Abs. 2 GG, $\S 46$ Abs. 2 BVerfGG zur (gesondert anzuordnenden) Auflösung der politischen Partei.

Die Zurückhaltung des Parteienrechts gegenüber politischen Parteien, die in ihren Satzungen und durch einzelne Beschlüsse solche gesetzlichen Bestimmungen verletzten, die der Sicherstellung ihrer demokratischen Binnenorganisation dienen, mag erneut als widersprüchlich verstanden werden, erscheint bei distanziertem Blick aber sinnvoll und richtig. Denn die bloße Parteieigenschaft ist rechtliche Voraussetzung für eine Reihe von Statusrechten und Privilegierungen, die politische Parteien gegenüber sonstigen Vereinigungen genießen. Der Entzug der Parteieigenschaft ist vor diesem Hintergrund ein schwerwiegender Eingriff in die Gründungs- und Betätigungsfreiheit sowie die formale Gleichheit politischer Parteien, der den staatlichen Institutionen (und den in ihnen vertretenen politischen Parteien) massive Missbrauchsmöglichkeiten eröffnet. Vor diesem Hintergrund ist der Umstand, dass vermeintlich - undemokratisch organisierte politische Parteien als solche weiterhin an der staatlichen Finanzierung politischer Parteien teilhaben, als geringeres Übel zu begreifen, zumal die Wähler die Art der internen Willensbildung - Transparenz vorausgesetzt ${ }^{25}$ - in ihrer Wahlentscheidung berücksichtigen können.

\section{Geltendmachung und Durchsetzung der Wirkungen}

Führen Verstöße von Satzungsbestimmungen gegen gesetzliche Vorschriften auch zu ihrer Nichtigkeit und sind auf der Grundlage nichtiger Satzungen oder unter Verletzung von Satzungsbestimmungen gefasste Beschlüsse auch unwirksam, stellt sich doch die Frage, wie diese rechtlichen Wirkungen geltend gemacht und durchgesetzt werden können. Ausgehend von der Feststellung, dass keine abstrakte Satzungskontrolle vorgesehen ist (1.), kommt insofern nur eine Inzidentkontrolle von Satzungen bei der Überprüfung konkreter Beschlüsse in Betracht (2.). Im Übrigen ist die Einhaltung der auf die innerparteiliche Willensbildung bezogenen rechtlichen Vorgaben nur einer politischen Kontrolle unterworfen (3.).

25 Dies betont G. Roellecke, in: Umbach/Clemens, GG, 2002, Art. 21 Rn. 72 ff,; vgl. auch $K$. Hesse, Die verfassungsrechtliche Stellung der politischen Partei im modernen Staat, VVDStRL 17, S. 11, 38. 


\section{Keine abstrakte Satzungskontrolle}

Obwohl § 6 PartG die Satzungsautonomie politischer Parteien zunächst in formaler Hinsicht dadurch beschränkt, dass die Satzung bestimmte obligatorische Mindestinhalte regeln muss, und die weiteren genannten Vorschriften das Gebot binnendemokratischer Organisation auch in inhaltlicher Hinsicht sichern wollen, kennt das Parteienrecht nur eine marginale staatliche Eröffnungskontrolle. Nach $\S 6$ Abs. 3 PartG muss der Vorstand der politischen Partei dem Bundeswahlleiter „1. Satzung und Programm der Partei, 2. Namen der Vorstandsmitglieder der Partei und der Landesverbände mit Angabe ihrer Funktionen und 3. (gegebenenfalls) die Auflösung der Partei oder eines Landesverbandes mitteilen." Dieser Mitteilungspflicht entspricht aber keine inhaltliche Prüfungsbefugnis des Bundeswahlleiters. Er kann und muss nur prüfen, ob die genannten Informationen mitgeteilt werden, darf diese Informationen aber nicht inhaltlich werten. ${ }^{26}$ Das ergibt sich aus Art. 38 Abs. 3 PartG. Danach kann der Bundeswahlleiter den Vorstand der Partei zur Vornahme der Handlungen nach $\S 6$ Abs. 3 PartG durch ein Zwangsgeld (zwischen 250 und $1.500 €$ ) anhalten. Seine Vollstreckungsbefugnis beschränkt sich aber ausdrücklich auf die Pflicht nach $\S 6$ Abs. 3 PartG, die materiellen Voraussetzungen des $\$ 6$ Abs. 2 PartG oder anderer Vorschriften sind nicht genannt. Der Grund für diese gesetzliche Zurückhaltung liegt in der verfassungsrechtlichen Gewährleistung der (Gründungs-)Freiheit der politischen Parteien, die durch eine wie auch immer geartete staatliche Eröffnungskontrolle mindestens gefährdet wenn nicht sogar schon verletzt wäre. Die Mitteilungspflicht des Vorstands gegenüber dem Bundeswahlleiter bewirkt somit explizit keine materielle Kontrollbefugnis des Bundeswahlleiters, sondem dient alleine der kontrollermöglichenden Publizität ${ }^{27}$ - die eingereichten Unterlagen können beim Bundeswahlleiter nach $\S 6$ Abs. 3 S. 3 PartG von jedermann eingesehen, kostenlose Abschriften nach $\S 6$ Abs. $3 \mathrm{~S}$. 4 PartG verlangt werden. Aus $\S 6$ Abs. 3 PartG lässt sich insoweit der Grundsatz entnehmen, dass die Kontrolle über Ausgestaltung und Einhaltung der rechtlichen Rahmenbedingungen für eine binnendemokratische Willensbildung der Öffentlichkeit obliegt, die typischerweise auf politische Sanktionsformen verwiesen ist.

\section{Inzidentkontrolle bei der Überprüfung konkreter Beschlüsse}

Ausnahmsweise kann es bei der Überprüfung konkreter Beschlüsse inzident auch zu einer Kontrolle der ihnen zu Grunde liegenden Satzungen kommen, nämlich durch den Bundeswahlleiter beim Verfahren der Kandidatenaufstellung (a) und im Rahmen von Gerichts- oder Schiedsgerichtsverfahren (b).

26 Vgl. S.-C. Lenski, in: Rossi/Lenski, PartG, §6 Rn. 43; S. Augsberg, in: Kersten/Rixen (Hrsg.), PartG, §6 Rn. 29; J. Ipsen, in: ders. (Hrsg.), ParteienG, § 6 Rn. 19; Kunig, in: HStR III, $\S 40 \mathrm{Rn} .36$.

27 Deutlich G. Roellecke, in: Umbach/Clemens, GG, 2002, Art. 21 Rn. 81. 
a) Kontrolle der Kandidatenaufstellung

Mit der Aufstellung von Parteibewerbern für die Parlamentswahlen ist der Schnittpunkt zwischen gesellschaftlicher Willensbildung und staatlicher Willensbetätigung berührt. Die Nominierung stellt insofern das Scharnier dar, an dem der Übergang von gesellschaftlich verwurzelter Parteipolitik in staatlich legitimierte Ausübung von Hoheitsgewalt stattfindet. Wegen dieser besonderen Bedeutung findet an dieser Stelle eine Kontrolle durch den Bundeswahlleiter statt, deren Umfang im Einzelnen umstritten ist. $^{28}$

Die Kontrolle beginnt mit einer Prüfung der Parteieigenschaft durch den Bundeswahlausschuss nach $\S 18$ Abs. 4 BWahlG und setzt sich mit der Prüfung der Kandidatenaufstellung durch den Bundeswahlleiter nach $\S 25 \mathrm{BWahlG}$ fort. Materieller Maßstab für diese Prüfung durch den Bundeswahlleiter sind die Vorgaben des $\S 21$ BWahlG, die ihrerseits gemäß $§ 21$ Abs. 5 BWahlG der Ausgestaltung durch die Satzungen der politischen Parteien vorbehalten sind. Daraus ergibt sich das grundsätzliche Problem, in welchem Umfang der Bundeswahlleiter die Aufstellung von Parteibewerbern prüfen darf und prüfen muss. Denn wenn etwa starre Blockwahlen $^{29}$ oder fixe Geschlechterquoten mit den Wahlrechtsgrundsätzen auch unvereinbar sind, ${ }^{30}$ sind die entsprechenden Satzungsbestimmungen und konkreten $\mathrm{Be}$ schlüsse doch wahlrechtlich grundsätzlich unerheblich. ${ }^{31}$ Dementsprechend kann ein solcher Verstoß weder zu einer Zurückweisung des Wahlvorschlags nach den $\$ \S 26$, $28 \mathrm{BWahlG}$ führen noch kann er taugliche Grundlage einer Wahlprüfungsbeschwerde sein. ${ }^{32}$ Verstöße gegen das Satzungsrecht bei der Aufstellung von Parteibewerbern können somit grundsätzlich nur innerparteilich gerügt werden, sei es vor den parteiinternen Schiedsgerichten, sei es (im einstweiligen Rechtsschutz) vor den ordentlichen Gerichten. In der Praxis scheint es hier häufig antizipierend zu informellen Gesprächen zwischen den politischen Parteien und dem Bundes- bzw. den Landeswahlleitern zu kommen. Dies ist so lange unbedenklich, wie die Wahlleiter nicht ein Wahlverfahren bei einer politischen Partei als zulässig tolerieren, das sie bei einer anderen für rechtswidrig halten.

Ausnahmsweise kann die parteiinterne Kandidatenaufstellung zwar nicht prospektiv, doch retrospektiv im Wahlprüfungsverfahren kontrolliert werden. So hat das Verfassungsgericht Hamburg die Wiederholung von Landtagswahlen angeordnet, weil nach Fehlern bei der Kandidatenaufstellung durch die CDU die Möglichkeit eines anderen Wahlverhaltens nicht ausgeschlossen werden konnte. ${ }^{33}$ Und das Bundesverfassungsgericht hat zu den (satzungsrechtlich konkretisierten) Rechten der Bewerber ausgeführt, dass „der zu den demokratischen Grundsätzen zählende

S. Augsberg, in: Kersten/Rixen (Hrsg.), PartG, $\$ 6$ Rn. 29; W. Schreiber, BWahlG, 8. Aufl. $2009, \S 25 \mathrm{Rn}$. I ff.

29 Vgl. hierzu BVerfGE 89, 243, 264.

30 S.-C. Lenski, in: Rossi/Lenski, PartG, \$21 BWahlG Rn. 82 ff.

31 BVerfGE 89, 243 (252).

32 S.-C. Lenski, in: Rossi/Lenski, PartG, $\$ 21$ BWahlG Rn. 87.

33 Hbg. VerfG. DVBI. 1993, S. 1072. 
Minderheitenschutz die Möglichkeit umfassen muss, sich persönlich vorzustellen und programmatische Aussagen machen zu können. ${ }^{.34}$

\section{b) Gerichts- und Schiedsgerichtsverfahren}

Eine Kontrolle der Übereinstimmung von Satzungen der Parteien mit den gesetzlichen Vorgaben sowie von Beschlüssen mit den Satzungen ist auch durch die parteiinternen Schiedsgerichte und die ordentlichen Gerichte möglich. Allerdings stehen der theoretischen Möglichkeit einer solchen Kontrolle zahlreiche rechtliche, darüber hinaus aber auch faktische Aspekte entgegen. In rechtlicher Hinsicht sei betont, dass die gerichtliche und auch die schiedsgerichtliche Kontrolle als individueller Rechtsschutz ausgestaltet ist. Dies manifestiert sich zunächst in entsprechenden Hürden der Zulässigkeit von Rechtsbehelfen. Ausreichend ist nicht schon jede (behauptete) objektive Verletzung der gesetzlichen Vorgaben durch die Satzung einer politischen Partei oder eben dieser durch einen konkreten Beschluss bzw. eine konkrete Personalentscheidung. Erforderlich ist vielmehr stets, dass eine Klagebefugnis im Sinne der Möglichkeit einer Verletzung eigener, subjektiver Rechte geltend gemacht werden kann. Im Ergebnis führt bereits diese Zulässigkeitsvoraussetzung zu einer Begrenzung der möglichen Klagegegenstände auf Personalentscheidungen - Sachentscheidungen oder bloße Verfahrensregelungen scheitern schon an der regelmäßig fehlenden Möglichkeit subjektiver Rechtsverletzungen. Zusätzlich eingeschränkt wird der Zugang zu Gerichten und Schiedsgerichten durch das Erfordernis des Rechtsschutzbedürfnisses. Und selbst wenn die Gerichte in eine tatsächliche Prüfung des Streitgegenstandes, in eine Begründetheitsprüfung, eintreten, ist ihre Prüfungsdichte mit Rücksicht auf die Freiheit der politischen Parteien und ihre Satzungsautonomie doch weit eingeschränkt. Im Ergebnis findet eine rechtliche Absicherung der innerparteilichen Willensbildung durch Gerichte und Schiedsgerichte deshalb kaum statt.

Was auf den ersten Blick als Rechtsschutzlücke verstanden werden kann, ist auf den zweiten Blick aber letztlich konsequent: ${ }^{35}$ Das Recht ist im politischen Prozess nur begrenzt wirksam, und mit der Bedeutung des Rechts schwindet auch die Bedeutung von Gerichten und Schiedsgerichten, die ausschließlich am Maßstab des Rechts entscheiden. Ihre theoretisch durchaus vorhandenen Möglichkeiten einer Rahmenkontrolle müssen zudem extern initiiert werden - mangels einer Selbstbefassungsbefugnis gilt nach wie vor: Wo kein Kläger, da kein Richter. Mögliche Kläger werden aber gerade im politischen Prozess viele Gründe haben, auf einen Rechtsschutz zu verzichten: Das Parteimitglied, das sich gegen eine Personalentscheidung seiner Partei erfolgreich in eine Vertreterversammlung zur Nominierung von Listenkandidaten einklagt und sich auf diesem Wahltag möglicherweise gar

35 Im Ergebnis auch $H$. Schiedermair, Parteiausschluss und gerichtlicher Rechtsschutz, in: AöR 104 (1979), S. 200 (223). 
selbst zur Wahl stellt, wird dort sicherlich keine große Erfolgsaussichten haben. Ein Schiedsgerichtsverfahren wird darüber hinaus nur einschlagen, wer auch etwas zu verlieren hat - was nur für Funktionäre und kaum für „einfache“ Parteimitglieder gilt. $^{36}$

\section{Politische Sicherung der binnendemokratischen Willensbildung}

Trotz dieser nüchternen Bilanz lässt sich nicht sagen, dass das Recht bei der Absicherung der normativen Anforderungen an eine binnendemokratische Organisation und innerparteiliche Willensbildung versagt. Es nimmt nur seinen Geltungsanspruch zurück und überlässt den Prozess der innerparteilichen Willensbildung und Entscheidungsfindung weitgehend dem Politischen. Damit ist auch die Kontrolle weitgehend eine politische, die ihrerseits aber vor allem auf Publizität angewiesen ist. Transparenz als Voraussetzung von Kontrollierbarkeit und Kontrolle gewinnt insoweit an besonderer Bedeutung. ${ }^{37}$ Als Akteure, die eine solche Kontrollierbarkeit in eine tatsächliche Kontrolle verwandeln können, kommen in erster Linie die Parteimitglieder in Betracht, denen nach $\S 10$ Abs. 2 S. 3 GG das Recht auf jederzeitigen Austritt und nach Art. 21 Abs. 1 S. 2 GG die Gründung einer neuen politischen Partei zusteht.

Darüber hinaus fungieren aber auch die Wähler als Kontrolleure. Denn wenn die Freiheit und Autonomie den politischen Parteien die Möglichkeit gibt, sich nicht nur durch Inhalte, sondern auch durch Organisationsformen zu profilieren, wird sich die innerparteiliche Willensbildung sowohl an den Interessen der Parteimitgliedern als auch an den (mutmaßlichen) Präferenzen der Wähler orientieren. Die Grünen etwa werden manchen Symphatisanten oder gar Wähler auch dadurch gewonnen haben, dass sie sich mehr als andere politische Parteien dem Grundsatz demokratischer Willensbildung und Entscheidungsfindung verschrieben haben, auch wenn manche vermeintlich besonders demokratische Verfahren der Sache nach schlicht rechtsund verfassungswidrig sind, wie etwa Rotationsverfahren ${ }^{38}$ oder starre Geschlechterquoten..$^{30}$

36 G. Roellecke, in: Umbach/Clemens, GG, 2002, Art. 21 Rn. 84; Ch. Gusy, in: AK-GG, 3. Aufl. 2001, Art. 21 Rn. 66.

37 Vgl. allgemein M. Rossi. Informationszugangsfreiheit und Verfassungsrecht. 2004, S. 77 f. u. $233 \mathrm{ff}$.

38 M. Morlok in: Dreier (Hrsg.), GG, Bd. II, 2. Aufl. 2006, Art. 38 Rn. 77; H.-H. Trute in: v. Münch/Kunig, Grundgesetz-Kommentar Art. 38 Rdnr. 32.

39 S.-C. Lenski, in: Rossi/Lenski. PartG, § 21 BWahlG, Rn. 82 fr.; vorsichtiger W. Schreiber, BWahlG. 8. Aufl. 2009, § 21 Rn. 39. 


\section{Erforderlichkeit und Zulässigkeit weiterer Sicherungsinstrumente?}

Soweit die rechtlichen Sicherungsinstrumente als defizitär empfunden werden (I.), können sich Überlegungen zu ihrer Stärkung auf die Erleichterung der Durchsetzung der bestehenden Wirkungen (II.) oder auf gänzlich neue Sanktionswirkungen beziehen, die freilich verfassungsrechtlichen Grenzen unterworfen sind (Ш.).

\section{Defizite rechtlicher Sicherungsinstrumente?}

Insbesondere mit Blick auf die Teilhabe an der staatlichen (Teil-)Finanzierung auch solcher politischer Parteien, deren Willensbildung und Entscheidungsfindung nach außen nicht besonders demokratisch erscheinen, werden die geringen rechtlichen Sanktionswirkungen mitunter als defizitär empfunden. Bereits seit den $60 \mathrm{er}$ Jahren des vergangenen Jahrhunderts gibt es deshalb Überlegungen, die innerparteiliche Willensbildung und Entscheidungsfindung weiter zu verrechtlichen. ${ }^{40}$ Wenn auch in diesem Beitrag den "Möglichkeiten" der rechtlichen Absicherung der innerparteilichen Willensbildung nachgegangen werden soll, kann insofern nicht nur das geltende Recht in den Blick genommen werden. De lege ferenda sind vielmeht eine ganze Reihe von Regelungen denkbar, der Phantasie andererseits aber auch juristische Grenzen gesetzt.

\section{Erleichterung der Durchsetzung der bestehenden Wirkungen}

Ausgehend von dem Befund, dass Verstöße gegen Bestimmungen, die die binnendemokratische Willensbildung und Entscheidungsfindung sicher stellen sollen, zwar theoretisch zur Nichtigkeit bzw. Unwirksamkeit der jeweiligen Rechtsakte führen, solche Verstöße aber häufig nicht festgestellt werden und die Sanktionswirkungen deshalb ausbleiben, beziehen sich erste Überlegungen im Sinne einer einfacheren politischen Umsetzbarkeit auf die Erleichtenung der Durchsetzung der bereits bestehenden Sanktionswirkungen. In Betracht kämen insofern ein präventiver Zulassungsvorbehalt für Satzungen (1.), die Einführung institutionalisierter Kontrollinstanzen (2.) oder ein Abbau von Zulässigkeitsvoraussetzungen (3.).

40 Ch. Gusy, in: AK-GG, 3. Aufl, 2001, Art. 21 Rn. 65, entnimmt dem Art. 21 Abs. 1 S. 3 GG aber sogar ein Verrechtlichungsgebot, weil die Bestimmung die Parteien überhaupt verpflichtet, sich eine Ordnung al geben. 


\section{Präventiver Zulassungsvorbehalt für Satzungen}

Zunächst ließe sich daran denken, die Satzungen der politischen Parteien einem Zulassungsvorbehalt und damit einer Prüfungsmöglichkeit und -befugnis staatlicher Stellen, bspw. des Bundeswahlleiters, zu unterstellen.

Eine solche Ausweitung von einer bloß formalen ${ }^{41}$ zu einer inhaltlichen Überprüfung von Satzungen ist der Rechtsordnung nicht fremd. Im Umweltrecht etwa sieht das Naturschutzrecht bestimmte verwaltungsverfahrensrechtliche und verwaltungsprozessuale Beteiligungsrechte von Vereinen vor, knüpft die Zuweisung dieser Rechte aber an die formale Anerkennung durch die jeweiligen Ministerien. Wenn $\S 59$ Abs. 1 Nr. 6 BNatSchG als materielle Voraussetzungen dabei u.a. normiert, dass jedermann einen Anspruch auf Zugang zu dem Verein und jedes Mitglied volles Stimmrecht haben muss, sind damit im Ansatz binnendemokratische Strukturen gefordert, wie sie über Art. 21 Abs. 1 S. 3 GG auch für politische Parteien gelten. Die für die skizzierten Rechte konstitutive Anerkennung von Vereinen setzt dabei die Prüfung voraus, dass diese satzungsmäßigen Rechte tatsächlich eingeräumt sind, und führt konsequent zur Verweigerung der Anerkennung, wenn Umweltschutzorganisationen trotz hehrer Zielsetzungen zwischen (quantitativ überwiegenden) Fördermitgliedern und wenigen, etwa nur 40 stimmberechtigten Mitgliedern unterscheiden, wie es etwa bei Geenpeace der Fall ist. ${ }^{42}$

Diese aus dem Umweltrecht, etwa aber auch dem Verbraucherschutzrecht bekannte formale und inhaltliche Prüfung von Vereinigungen, die einer Anerkennung und mit ihr einer Gewährung bestimmter subjektiver Rechte vorausgeht, darf aber nicht auf politische Parteien übertragen werden. Dem steht der Unterschied zwischen politischen Parteien auf der einen Seite und bestimmten Interessen verschriebenen Vereinen auf der anderen Seite entgegen, der verfassungsrechtlich durch die Privilegierung von politischen Parteien in Art. 21 GG gegenüber der rechtlichen Behandlung von sonstigen Vereinen nach Art. 9 GG zum Ausdruck kommt. Dieser Unterschied manifestiert sich ungeachtet zahlreicher Einzelheiten vor allem darin, dass politische Parteien primär auf die (gesamte) politische Willensbildung im Staate durch eine (im besten Falle erfolgreiche) Beteiligung an Wahlen zu Volksvertretungen mitwirken wollen, in denen sie bzw. ihre Vertreter sodann Einfluss auf die Rechtsetzung nehmen, während sonstige Vereine in erster Linie auf eine Beteiligung im exekutiven Bereich zielen. Mögen die Grenzen zwischen exekutivem und legislativem Handeln insbesondere auch dort verschwimmen, wo der Exekutive mit Planungsaufgaben zahlreiche Gestaltungsmöglichkeiten übertragen sind, bleibt der institutionelle Unterschied zwischen beiden Formen staatlicher Steuerung in einer repräsentativen Demokratie doch erheblich. Insofern steht einer inhaltlichen Zulassungskontrolle von Satzungen oder gar Programmen die verfassungsrechtlich garan-

41 S. Augsberg, in: Kersten/Rixen (Hrsg.). PartG, verneint in $\$ 6 \mathrm{Rn} .30$ schon ein formelles Prüfungsrecht.

42 Vgl. \$ 4 Satzung des Geenpeace e.V. 
tierte Gründungs- und Betätigungsfreiheit der politischen Parteien entgegen, der eine strikte Neutralitätspflicht des Staates entspricht. ${ }^{43}$

\section{Institutionalisierte Kontrollinstanzen}

Zur Durchsetzung der bestehenden rechtlichen Regelungen, die der Sicherung der binnendemokratischen Organisation politischer Parteien dienen, könnten institutionalisierte, „objektive“ Kontrollinstanzen geschaffen werden. Sie verringerten das faktische Problem, dass es in vielen Fällen mangels Kläger keinen Richter gibt. Gedacht wird etwa an eine Art Kartellbehörde für den politischen Wettbewerb, aber auch die Einsetzung von Ombudsmännern oder Beauftragten wäre denkbar. Milder noch und im Trend der regulierten Selbstregulierung könnte auch ein Gütesiegel für Satzungen politischer Parteien entwickelt werden, das nach einer Evaluierung der binnendemokratischen Entscheidungsfindung von einer staatlich beauftragten, jedoch privat ausgestalteten Evaluierungsstelle vergeben würde, die dann wahrscheinlich paritätisch von allen oder jedenfalls den in einem deutschen Parlament vertretenen politischen Parteien zu besetzen wäre.

All solchen Überlegungen ist aber entschieden entgegenzutreten. Es ist weder sinnvoll noch mit Blick auf die verfassungsrechtlich begründete Freiheit der politischen Parteien zulässig, staatliche Sicherungsinstrumente - wie mittelbar sie auch ausgestaltet sein mögen - in die Parteien hineinzutragen. Im pluralistischen Parteiensystem sind politische Parteien Vereinigungen, die im gesellschaftlichen Bereich gründen und bei der politischen Willensbildung des Volkes mitwirken. Diese Willensbildung soll nicht durch den Staat gelenkt und antizipiert werden. Jede vermeintlich „objektive“ Kontrollinstanz führte zu einer Verrechtlichung des Parteiwesens, die damit dem staatlichen Einfluss ausgesetzt wäre. Dies führte zwangsläufig zu einer Herrschaft der etablierten politischen Parteien, erschwerte die Gründung neuer politischer Parteien und widerspräche insofern letztlich sogar dem Demokratieprinzip, das Herrschaft nur auf Zeit und Minderheiten die Möglichkeit einräumt, zur Mehrheit zu werden.

\section{Abbau der prozessualen Zulässigkeitsvoraussetzungen}

Schließlich könnte den bestehenden rechtlichen Sicherungsbestimmungen dadurch zu mehr Wirksamkeit verholfen werden, dass die Zulässigkeitsvoraussetzungen für die Einleitung eines Schieds- oder Gerichtsverfahrens abgesenkt werden. In gewissem Maße führte etwa der Verzicht auf das Erfordemis einer Klagebefugnis zu einer Objektivierung der Klage, ohne jedoch eine Institutionalisierung und damit die Ge- 
fahr einer jedenfalls mittelbaren staatlichen Einflussnahme zu bewirken. Die Klageberechtigung könnte einerseits von dem Erfordernis der möglichen Verletzung eigener Rechte befreit, andererseits aber doch etwa auf Parteimitglieder beschränkt werden. Solche und andere differenzierende Lösungen mögen sinnvoll und wohl auch verfassungsrechtlich zulässig sein, stünden aber - jedenfalls in Bezug auf Personalentscheidungen - stets vor der Schwierigkeit, dass nur ein rechtzeitiger Rechtsschutz effektiver Rechtsschutz ist. Will man aber nicht zugleich die rechtsstaatlich und z.T. auch grundrechtlich begründeten Anforderungen an Gerichtsverfahren absenken, käme Rechtsschutz etwa gegen die Nominierung von bestimmten Kandidaten oder gegen sonstige Personalentscheidungen entweder zu spät oder blockierte im Wege einstweiliger Anordnungen die Entscheidungsfindung. Außerdem darf nicht übersehen werden, dass, jedenfalls bei der Anrufung der staatlichen Gerichte, der Staat erneut in erheblicher Weise Einfluss nehmen könnte auf die Willensbildung und Entscheidungsfindung in politischen Parteien. Zwar wiegte dieser Eingriff weniger schwer als solche der Exckutive, weil Richter in besonderer Weise ausgebildet und gegenüber den anderen Gewalten durch Art. 97 Abs. 1 GG mit einer besonderen Unabhängigkeit geschützt sind. Doch bliebe die Gefahr, dass nicht nur das Parteiwesen zunehmend verrechtlicht würde, was noch als positiv bewertet werden mag, sondern vor allem auch, dass umgekehrt das Gerichtswesen zunehmend parteipolitisiert würde. Insofern scheint die Balance zwischen primär politischer und begrenzt rechtlicher Kontrolle der rechtlichen Anforderungen an eine binnendemokratische Organisationsstruktur der politischen Parteien im geltenden Recht durchaus gefunden zu sein.

\section{Mögliche zusätzliche rechtliche Sanktionen}

Vor diesem Hintergrund erscheinen auch Überlegungen zur Einführung neuer rechtlicher Sanktionswirkungen, die über die Effektivierung der bereits bestehenden Sanktionen hinausgehen, eher als Gedankenspiele. Solche Überlegungen sind nicht neu, sondern wurden bspw. intensiv schon in den 70er Jahren des vergangenen Jahrhunderts geführt. ${ }^{44}$ Insbesondere zwei Vorschläge werden immer wieder unterbreitet: Die Missachtung binnendemokratischer Grundsätze solle entweder zum Verlust der staatlichen Finanzierung (1.) oder gar zum Ausschluss von der Teilnahme an Wahlen führen (2.).

44 Vgl. insb. $R$. Wolfrum, Die innerparteiliche Ordnung nach dem Parteiengesetz, Berlin 1974. passim. 
Die Unterstützung einer politischen Partei, die gegen das Gebot innerparteilicher Demokratie verstößt, aus Steuermitteln wird zum Teil als unbefriedigend empfunden. Sie von der staatlichen Finanzierung auszuschließen, sei insofern ein adäquates Sanktionsmittel. ${ }^{45}$

Die Überlegung erscheint in sich durchaus konsequent. Auf der Grundlage des geltenden Rechts dürften entsprechende Sanktionen aber nicht verhängt werden. Selbst die Befüworter erkennen, dass die Verweigerung von staatlichen Mitteln an eine politische Partei eine wesentliche Beeinträchtigung ihrer Wettbewerbschancen und somit einen Eingriff in die Gleichheit der politischen Parteien darstellt, der einer gesetzlichen Grundlage bedarf. ${ }^{46}$ Doch unabhängig von einem Gesetzesvorbehalt wird schon die generelle Zulässigkeit solcher Sanktionen bestritten. ${ }^{47}$ Art. 21 Abs. 2 GG normiere die einzige staatliche Sanktionsmöglichkeit und entfalte Sperrwirkung gegenüber anderen möglichen Sanktionen. ${ }^{48}$

Dieses Argument ist beachtlich und schlägt im Ergebnis durch. Die Freiheit der politischen Parteien und insbesondere auch ihre Chancengleichheit lassen es nicht zu, dass der Staat gesetzliche Sanktionsmöglichkeiten schafft und sich unter Ausnutzung seiner Interpretationshoheit über die gesetzlich eingeführten Tatbestandsmerkmale in die Lage versetzt, bestimmte politische Parteien von der staatlichen Finanzierung auszuschließen.

Demgegenüber verfängt das Argument, dass doch auch unrichtige Rechenschaftsberichte nach $\S 31$ a PartG zu einem Ausschluss von der Parteienfinanzierung führen können, aus zwei Gründen nicht. Erstens besteht zwischen den Rechenschaftsberichten der politischen Parteien und den Festsetzungen der staatlichen Finanzierung ein enger sachlicher Zusammenhang, der zwischen der binnendemokratischen Organisation und der staatlichen Finanzierung nicht erkennbar ist. Im ersten Fall bestimmen die Angaben über die eigenen Einnahmen der Partei nach $\$ 19$ Abs. 5 PartG unmittelbar auch den Anteil an der staatlichen Finanzierung, im zweiten Fall würde die Verweigerung staatlicher Zahlen allein straf- oder strafähnlichen Charakter haben. Zweitens und vor allem aber darf nicht übersehen werden, dass selbst politische Parteien, die die materiellen Voraussetzungen des Art. 21 Abs. 2 S. 1 GG erfüllen, bis zu einer entsprechenden Feststellung des Bundesverfassungsgerichts an der staatlichen Finanzierung partizipieren. Insofern entfaltet Art. 21 Abs. 2 GG sehr wohl Sperrwirkung nicht nur für Sanktionen, die auf die Abwehr von Gefahren für die politische Ordnung in der Bundesrepublik Deutschland abzielen, sondern auch für solche, die an die - vermeintlich - undemokratische Binnenstruktur anknüpfen.

M. Morlok, in: Dreier (Hrsg.), GG-Kommentar, Bd. 2, 2. Aufl. 2006, Art. 21 Rn. 128 m.w.N. M. Morlok, in: Dreier (Hrsg.), GG-Kommentar, Bd. 2, 2. Aufl. 2006, Art. 21 Rn. 128. Vgl. insb. H.-H. Klein, in: Maunz Dürig/(Hrsg.), GG, Art. 21 Rn. 358.

A.A. K. Hesse, Die verfassungsrechtliche Stellung der politischen Partei im modernen Staat. VVDStRL 17, S. 11, 29. 
Freilich darf mit Blick auf die tatsächliche Entscheidungsfindung in den politischen Parteien etwas zynisch vermutet werden, dass von einer gesetzlichen Möglichkeit des Ausschlusses politischer Parteien von der staatlichen Finanzierung im Ergebnis wohl alle Parteien betroffen wären, so dass ein solches Sanktionsmittel vielleicht zur Beendigung sämtlicher staatlicher Parteienfinanzierung führen würde.

Unabhängig von solchen Überlegungen bliebe das Problem, vor dem Hintergrund solcher scharfen Sanktionen den Maßstab binnendemokratischer Organisation noch bestimmter fassen zu müssen, ohne zugleich in die Satzungsautonomie der politischen Parteien einzugreifen. Zugleich müssten insbesondere auch das Verfahren zur Feststellung von undemokratischen Organisationsformen gesetzlich ausdifferenziert und die Zuständigkeit einer Stelle begründet werden, die ihrerseits unabhängig von den überkommenen staatlichen Akteuren handeln müsste. Alles in allem überwiegen deshalb die verfassungsrechtlichen Bedenken gegen die Einführung eines solchen Sanktionsmittels.

\section{Ausschluss von der Teilnahme an Wahlen}

Gleiches gilt in noch schärferem Maße für den Ausschluss von der Teilnahme an Wahlen. Politische Parteien sind ihrer Definition nach darauf aus, an Wahlen zu Volksvertretungen im Bund oder in Ländern teilzunehmen und die gesellschaftliche Willensbildung auf diese Weise in die staatlichen Organe zu tragen. Sie von dieser Möglichkeit auszunehmen, weil sie nicht den rechtlichen Anforderungen an eine binnendemokratische Organisationsform entsprechen, die ihrerseits verfassungsrechtlich nur vage normiert sind und auch gesetzlich nicht hinreichend bestimmt werden können, ohne zugleich die Freiheit und die erwünschte Pluralität politischer Parteien zu gefährden, wäre ein Schritt zur Etablierung von Staatsparteien, die mit der Verfassungsordnung des Grundgesetzes nicht vereinbar sind. ${ }^{49}$

\section{E. Fazit}

Politische Parteien sind privatrechtliche Vereinigungen, die sich im Rahmen der Privatautonomie gestalten. Sie entscheiden selbst über die Ausgestaltung demokratischer Grundsätze und über das $\mathrm{Maß}$ basisdemokratischer Entscheidungsfindung.

Dass der Staat rechtliche und rechtsstaatliche Mindestanforderungen an die binnendemokratische Organisation stellt, ohne umfassende Sanktionen für den Fall ihrer Missachtung bereit zu halten, ist kein Widerspruch. Vielmehr entspricht es der besonderen Stellung und Aufgaben der politischen Parteien zwischen Gesellschaft

49 Vgl. D. Grimm, in: Schneider/Zeh (Hrsg.), Parlamentsrecht und Parlamentspraxis in der Bundesrepublik Deutschland, 1989, §6 Rn. 11: „Die pluaralistische Demokratie kennt keine Staatspartei.“ 
und Staat, wenn der Staat Mindestanforderungen an die Organisation und Entscheidungsfindung in den politischen Parteien normiert, ihre Durchsetzung aber weitgehend den Mitgliedern der Partei und den Wählern überlässt. Die Art und Weise der Willensbildung gehört somit zu den Elementen, mit denen die politischen Parteien um die Gunst der Bürger streiten können. Geschicktes Marketing mag solche Elemente hervorheben und andere kaschieren, wird sich aber stets auch an der Nachfrage, dem Phantom des Wählerwillens, orientieren. Insofern werden die Wähler vielleicht solche Parteien favorisieren, in denen demokratische Entscheidungsfindung bis hin zur weitgehenden Einbindung direkt-demokratischer Elemente ${ }^{50}$ hoch gehalten wird. Vielleicht stimmen die Wähler aber auch für die Kandidaten solcher Parteien, die nach außen geschlossen auftreten, unabhängig davon, wie innerhalb der Parteien die Beschlüsse gefasst werden. Die Ambivalenz, der sich die politischen Parteien in ihren Marketingstrategien hinsichtlich ihrer Mitglieder auf der einen Seite und ihrer Wähler auf der anderen Seite gegenüber sehen, die zwischen dem verständlichen Wunsch nach Überzeugungshomogenität, Tendenzreinheit und Geschlossenheit einerseits und der rechtlichen Forderung nach einer demokratischen Organisationsstruktur andererseits besteht, kann durch rechtliche Regelungen nicht aufgelöst werden. 